\title{
Prasugrel Inappropriate Use in Patients Post-percutaneous coronary intervention (PCI). A Single Center Study
}

Ahmed Amro, MD' ${ }^{1}$, Kanaan Mansoor, MD', Mohammad Amro, MD², Amal Sobeih, MD³, Obadah Aqtash, MD' ${ }^{1}$, Hisham Hirzallah, MD ${ }^{1}$, Alaa Gabi, MD1', Madhulika Urella, MD' ${ }^{1}$, Waseem Ahmed, MD¹, Sandra Shenouda, MD ${ }^{1}$, Rameez Sayyed, MD

\section{ABSTRACI}

Prasugrel is a thienopyridine that was approved by the US Food and Drug Administration in combination with aspirin for the reduction of thrombotic events as well as stent thrombosis in patients with acute coronary syndrome who undergo percutaneous coronary intervention. This retrospective study aims to assess the frequency of inappropriate use of prasugrel and to emphasize that prasugrel still needs more attention as inappropriate use may result in significant morbidity.
Author affiliations are listed at the end of this article.

Correspondence to: Ahmed Amro, MD Marshall University Joan C. Edwards School of Medicine amro@marshall.edu

\section{KEYWORDS}

prasugrel, inappropriate use, coronary intervention, DAPT

\section{BACKGROUND}

The cornerstone in the management of acute coronary syndrome (ACS) is dual antiplatelet therapy (DAPT) including aspirin and P2Y12 inhibitors. ${ }^{1}$ DAPT is most beneficial for patients undergoing percutaneous coronary intervention $(\mathrm{PCl})$; it is known to reduce the occurrence of stent thrombosis and major cardiac events. ${ }^{2-4}$ Clopidogrel is the most commonly used P2Y12 inhibitor. Limitations of clopidogrel include variable antiplatelet effect ${ }^{5}$ and delayed onset of action. ${ }^{3}$ This variability in the efficacy of clopidogrel paved the way for newer P2Y12 inhibitors such as prasugrel to be used in selective cases.

Prasugrel is a thienopyridine that was approved by the US Food and Drug Administration (FDA) in combination with aspirin for the reduction of thrombotic events as well as stent thrombosis in patients with ACS who undergo $\mathrm{PCl}$. As prasugrel is a prodrug, it requires conversion to an active metabolite before binding to the P2Y12 receptor. ${ }^{6}$ TRITON-TIMI 38 was a randomized clinical trial which compared prasugrel and clopidogrel in patients with ACS and concluded that prasugrel (in comparison to clopidogrel) has an early onset of action and provides significant inhibition of platelet aggregation in patients with $A C S, 7,8$ thereby significantly reducing rate of stent thrombosis and ischemic events in patient undergoing $\mathrm{PCl}$. While there is an obvious benefit over clopidogrel, prasugrel is also associated with increased risk for major bleeding. ${ }^{8}$ This risk of bleeding led to the conclusion that there is no net benefit of prasugrel in patients with previous stroke or transient ischemic attack (TIA), patients older than 75 years, and patients weighing $<60 \mathrm{~kg}$; it was concluded that it caused net harm..$^{8-10}$ For these reasons, use of prasugrel in such patient populations is a class III recommendation. ${ }^{1}$

This retrospective study aims to assess the frequency of inappropriate use of prasugrel and to emphasize that prasugrel still needs more attention as inappropriate use may result in significant morbidity. Methods

StUdY DESIGN AND PATIENT POPULATION 
This is a single center retrospective study to determine the inappropriate use of prasugrel in patients during the period between July 2014 and July 2015 . Use of prasugrel in patients with history of cerebrovascular disease (CVA) or history of bleed or in patients who weigh $<60$ $\mathrm{kg}$, or patient with age of $\geq 75$ years was deemed as inappropriate. No exclusion criteria were applied. Marshall University's institutional review board approved the study.

\section{ENDPOINTS}

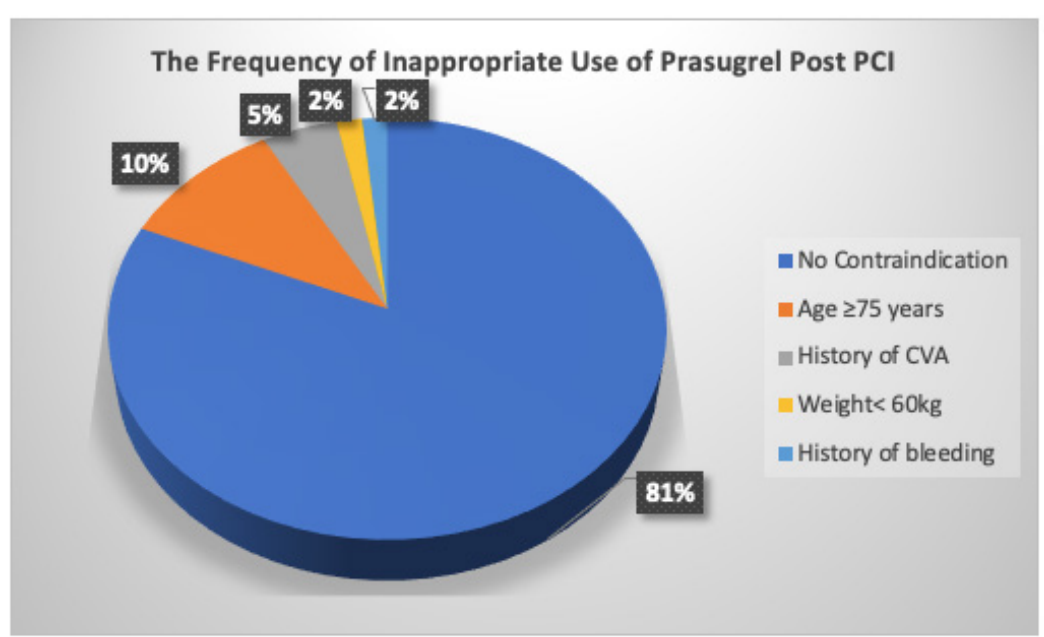

The primary study endpoint was the number of patients discharged to home on prasugrel. Secondary endpoints included patients prescribed prasugrel who had a history of CVA or bleed, weighed $<60 \mathrm{~kg}$, or were of age $\geq 75$ years.

\section{Data collection}

Patient's clinical characteristics, demographics, as well as primary and secondary endpoints, were retrospectively collected from the patient's electronic medical records.

\section{Statistical ANALYSIS}

Categorical variables were presented as percentages.

\section{RESULTS}

Duration of this study was July 2014 to July 2015. A total of 937 patients had $\mathrm{PCl}$ during this time; prasugrel was prescribed to 124 (12.3\%). Prasugrel was inappropriately used in $18.5 \%(n=23)$ of patients. Of these patients, $4.8 \%(n=6)$ had a history of CVA, $1.6 \%(n=2)$ had a history of bleeding, $10.5 \%(n=13)$ were aged $\geq 75$ years and $1.6 \%(n=2)$ weighed less than $60 \mathrm{~kg}$ (Chart 1).

\begin{tabular}{|l|c|}
\hline Age $(\mathbf{7 5}$ years) & Patient Discharge on Prasugrel (n=124) \\
\hline Male (n) & $13(10.5 \%)$ \\
\hline Female (n) & $92(74.2 \%)$ \\
\hline Weight/kg (<6) & $32(25.8 \%)$ \\
\hline TIA/Stroke (n) & $2(1.6 \%)$ \\
\hline HTN (n) & $6(4.8 \%)$ \\
\hline History of bleed & $101(81.5 \%)$ \\
\hline DM (n) & $2(1.6 \%)$ \\
\hline STEMI (n) & $52(41.9 \%)$ \\
\hline NSTEMI (n) & $21(16.9 \%)$ \\
\hline Unstable Angina (n) & $30(24.2 \%)$ \\
\hline $\begin{array}{l}\text { Elective Procedure } \\
\text { (n) }\end{array}$ & $56(45.2 \%)$ \\
\hline CAD (n) & $17(13.7 \%)$ \\
\hline Stent (n) & $74(59.7 \%)$ \\
\hline No stent (n) & $120(96.8 \%)$ \\
\hline
\end{tabular}

Our data demonstrated that prasugrel was also

TABLE 1: Demographics of patients who received prasugrel.

used for patients who did not receive stents during their procedure; the incidence was calculated as $3.2 \%(n=4)$. Among patients who were prescribed prasugrel, $16.9 \%(n=21)$ presented with ST-elevation myocardial infarction, $24.2 \%(n=30)$ presented with non-ST elevation myocardial infarction, while $45.2 \%$ $(n=56)$ patients had unstable angina and $13.7 \%$ $(\mathrm{n}=17)$ patients received prasugrel after elective $\mathrm{PCl}$ (Table 1). 


\section{DISCUSSION}

As stated in the 2016 update of the American College of Cardiology/American Heart Association DAPT guidelines prasugrel can only be used in place of clopidogrel in patients with ACS if the patient is not at an increased risk of bleeding; this is a Class IIB recommendation. 1 This recommendation was based on the findings of the TRITON-TIMI 38 trial, which concluded that prasugrel significantly decreases ischemic complications of ACS in patients undergoing $\mathrm{PCl}$ in comparison to clopidogrel.11 The same study also found that prasugrel, when used as a part of DAPT in a patient with a history of cerebrovascular event, patients aged 75 or older, and those weighing $<60 \mathrm{~kg}$, had increased rate of fatal bleeding. The aim of our study was to report the prevalence of inappropriate use of prasugrel.

Our study demonstrates that the inappropriate use of prasugrel is significant and it affects a sizable portion of the patients requiring antiplatelet therapy. Secondly, we were able to deduce that the inappropriate use of prasugrel most frequently occurs when it is prescribed to patients aged $>75$ years; in our sample it was a staggering $10.5 \%$. Alexopoulos et al., reported that age $\geq 75$ years is a common inappropriate use of $\mathrm{P} 2 \mathrm{Y} 12$ inhibitor (not limited to prasugrel). ${ }^{12}$ It would be noteworthy to mention that the rate of bleeding requiring transfusion was found to be significantly higher for patients receiving prasugrel inappropriately as compared with those receiving the drug under no contraindications. ${ }^{13}$

Alexopoulos et al. studied the Greek antiplatelet registry (GRAPE) and reported that $10.6 \%$ of the patients were inappropriately prescribed prasugrel on discharge. ${ }^{12}$ In comparison to the GRAPE registry, our data shows that $18.5 \%$ patients were prescribed prasugrel inappropriately. Hira et al. studied PINNACLE national registry and found that $13.9 \%$ patients were inappropriately prescribed prasugrel while $4.4 \%$ patients were prescribed prasugrel for non-recommended indications; ${ }^{14}$ the cumulative use was $18.3 \%$ which is consistent with findings of our study.

Variability in the prevalence of inappropriate use of prasugrel in the above-mentioned studies can be attributed to the difference in definition of "inappropriate use." Randomized studies that established the value of the P2Y12 inhibitors in the treatment of ACS patients undergoing $\mathrm{PCl}$ had excluded patients with many of the contraindications and special warnings mainly because of the accompanying increased risk of bleeding, without reporting on the prevalence of these characteristics in screened patients. ${ }^{15}$ Such clinical research practice led to masking of valuable information about the population that is possibly affected the most by inappropriate use of prasugrel. Secondly, the definition of our study does not differentiate between non-recommended use or inappropriate use. Hence, our data reports a higher rate of inappropriate use.

Prasugrel was also used in patients who had an elective $\mathrm{PCl}$, the proportion of such patients was $13.7 \%$ of our data. Use of prasugrel in this capacity has not been studied in detail. ${ }^{16}$ Marchini et al. devised an algorithm for prasugrel use in 2010 but concluded that the use of prasugrel might be considered for patients where the coronary artery anatomy is "high-risk" and patients who have homozygous reduced-function CYP allele which renders clopidogrel hypo-responsive. ${ }^{16}$ In 2012, TRIGGER-PCI study by Trenk et al. compared prasugrel and clopidogrel after elective $\mathrm{PCl}$, and found that $\mathrm{P} 2 \mathrm{Y} 12$ reaction unit (PRU) was significantly reduced in the prasugrel arm of the study. The study, however, could not demonstrate the benefit of prescribing prasugrel over clopidogrel in terms of endpoints which included cardiac death and myocardial infarction in six months. ${ }^{17}$

Damman et al. in 2014 reported that in respect to elective $\mathrm{PCl}$, occurrence of mortality and bleeding was comparable between the clopidogrel group and the prasugrel group. ${ }^{18} \mathrm{~A}$ recent randomized clinical trial by Hochholzer et al. "The ExcelsiorLOAD Trail" which compared P2Y12 inhibitors pharmacodynamics in peri interventional setting, reported that though prasugrel is more effective, the prasugrel arm of the study had a higher incidence of bleeding events. ${ }^{19}$ Earlier in 2013, European Society of Cardiology recommended that prasugrel should only be used in high-risk patients who require elective stenting. ${ }^{20}$ Use of prasugrel in elective $\mathrm{PCl}$ patients is still under debate and would need further 
studies to establish guidelines.

Literature review reveals that prasugrel use is gradually increasing with time, but it is accompanied with its inappropriate use. ${ }^{4,5}$ Therefore, physicians should use caution when prescribing prasugrel to patients undergoing $\mathrm{PCl}$ as inappropriate use may result in significant morbidity. Further studies assessing methods to overcome inappropriate prescribing of prasugrel would provide models for improvement.

The prasugrel package insert's black box warning recommends against its use in patient population with a history of cerebrovascular events, in patients aged 75 or older, and in patients weighing $60 \mathrm{~kg}$ or less. Despite these measures, evidence dictates that there has been an increase in the momentum of inappropriate or non-recommended use of prasugrel. Firstly, we strongly encourage caution in using prasugrel for patients who undergo elective PCls. Prasugrel may have its benefits but judicious approach by the physician is warranted to avoid bleeding events. Secondly, it is also needful to emphasize that patients aged $>75$ are at significantly increased risk of morbidity and mortality, if there are prescribed prasugrel.

\section{CONCLUSIONS}

According to our results, despite many years of FDA approval and well-known contraindications, prasugrel use in patients with known contraindications is not uncommon.

\section{AUTHOR AFFILIATIONS}

1. Marshall University Joan C. Edwards School of Medicine, Huntington, West Virginia

2. School of Medicine, MUST - Misr University for Science \& Technology, Cairo, Egypt

3. School of Medicine, Al-Najah University, Nablus, Palestine

\section{REFERENCES}

1. Levine GN et al. 2016 ACC/AHA Guideline Focused Update on Duration of Dual Antiplatelet Therapy in Patients With Coronary Artery Disease: A Report of the American College of Cardiology/American Heart Association Task Force on Clinical Practice Guidelines: An Update of the 2011 ACCF/AHA/SCAI Guideline for Percutaneous Coronary Intervention, 2011 ACCF/AHA Guideline for Coronary Artery Bypass Graft Surgery, 2012 ACC/AHA/ACP/AATS/PCNA/ SCAI/STS Guideline for the Diagnosis and Management of Patients With Stable Ischemic Heart Disease, 2013 ACCF/AHA Guideline for the Management of ST-Elevation Myocardial Infarction, 2014 AHA/ACC Guideline for the Management of Patients With Non-ST-Elevation Acute Coronary Syndromes, and 2014 ACC/ AHA Guideline on Perioperative Cardiovascular Evaluation and Management of Patients Undergoing Noncardiac Surgery. Circulation, 2016;134(10):e123-55.

2. Mehta SR, Yusuf S. Short- and long-term oral antiplatelet therapy in acute coronary syndromes and percutaneous coronary intervention. J Am Coll Cardiol. 2003;41 (4 Suppl S):79s-88s.

3. Steinhubl SR et al. Early and sustained dual oral antiplatelet therapy following percutaneous coronary intervention: a randomized controlled trial. Jama. 2002;288(19):2411-2420.

4. Yusuf $\mathrm{S}$ et al. Effects of clopidogrel in addition to aspirin in patients with acute coronary syndromes without ST-segment elevation. N Engl J Med 2001;345(7):494-502.

5. Serebruany VL et al. Variability in platelet responsiveness to clopidogrel among 544 individuals. J Am Coll Cardiol. 2005;45(2):246-51.

6. Niitsu Y et al. Pharmacology of CS-747 (prasugrel, LY640315), a novel, potent antiplatelet agent with in vivo $\mathrm{P} 2 \mathrm{Y} 12$ receptor antagonist activity. Semin Thromb Hemost. 2005;31(2):184-94.

7. Kohli $P$ et al.,Discharge aspirin dose and clinical outcomes in patients with acute coronary syndromes treated with prasugrel versus clopidogrel: an analysis from the TRITON-TIMI 38 study (trial to assess improvement in therapeutic outcomes by optimizing platelet inhibition with prasugrel-thrombolysis in myocardial infarction 
38). J Am Coll Cardiol. 2014;63(3):225-32.

8. Nijjer SS, Davies JE, Francis DP. Quantitative comparison of clopidogrel $600 \mathrm{mg}$, prasugrel and ticagrelor, against clopidogrel $300 \mathrm{mg}$ on major adverse cardiovascular events and bleeding in coronary stenting: synthesis of CURRENTOASIS-7, TRITON-TIMI-38 and PLATO. Int J Cardiol. 2012;158(2):181-185.

9. De Servi $\mathrm{S}$ et al. Prasugrel and ticagrelor compared to clopidogrel in non-ST-segment elevation acute coronary syndromes undergoing percutaneous coronary interventions: Certainties and uncertainties. Int J Cardiol. 2015;181:443-445.

10. Greenhalgh J et al. Prasugrel (Efient(R)) with percutaneous coronary intervention for treating acute coronary syndromes (review of TA182): systematic review and economic analysis. Health Technol Assess. 2015;19(29):1-130.

11. Wiviott SD et al. Prasugrel versus clopidogrel in patients with acute coronary syndromes. N Engl J Med. 2007;357(20):2001-2015.

12. Alexopoulos $D$ et al. Implementation of contemporary oral antiplatelet treatment guidelines in patients with acute coronary syndrome undergoing percutaneous coronary intervention: a report from the GReek AntiPlatelet rEgistry (GRAPE). Int J Cardiol. 2013;168(6):53295335.

13. Sandhu A et al. Contemporary use of prasugrel in clinical practice: insights from the Blue Cross Blue Shield of Michigan Cardiovascular Consortium. Circ Cardiovasc Qual Outcomes 2013;6(3):293298.

14. Hira RS et al. Frequency and practice-level variation in inappropriate and nonrecommended prasugrel prescribing: insights from the NCDR PINNACLE registry. J Am Coll Cardiol. 2014;63(25 Pt A): 2876-2877.

15. Wallentin $L$ et al. Ticagrelor versus clopidogrel in patients with acute coronary syndromes. N Engl J Med. 2009;361(11):1045-1057.

16. Marchini J et al. An algorithm for use of prasugrel (effient) in patients undergoing cardiac catheterization and percutaneous coronary intervention. Crit Pathw Cardiol. 2010;9(4):192198.

17. Trenk $D$ et al. A randomized trial of prasugrel versus clopidogrel in patients with high platelet reactivity on clopidogrel after elective percutaneous coronary intervention with implantation of drug-eluting stents: results of the TRIGGER-PCI (Testing Platelet Reactivity In Patients Undergoing Elective Stent Placement on Clopidogrel to Guide Alternative Therapy With Prasugrel) study. J Am Coll Cardiol 2012;59(24): 2159-2164.

18. Damman $P$ et al. Treatment patterns and outcomes in patients undergoing percutaneous coronary intervention treated with prasugrel or clopidogrel (from the Swedish Coronary Angiography and Angioplasty Registry [SCAAR]). Am J Cardiol. 2014;113(1):64-69.

19. Hochholzer W et al. Randomized comparison of different thienopyridine loading strategies in patients undergoing elective coronary intervention: The ExcelsiorLOAD Trial. JACC Cardiovasc Interv. 2016;9(3): 219-227.

20. Task Force Members et al. 2013 ESC guidelines on the management of stable coronary artery disease: the task force on the management of stable coronary artery disease of the European Society of Cardiology. Eur Heart J. 2013;34(38):2949-3003. 